\title{
OBSERVATIONS OF STRONG STELLAR LINES WITH THE OAO
}

\author{
A. D. CODE and R. C. BLESS \\ Space Astronomy Laboratory, Washburn Observatory, \\ University of Wisconsin, Madison, Wis., U.S.A.
}

\begin{abstract}
This paper reports on preliminary analysis of spectral scans of early-type stars obtained with the Orbiting Astronomical Observatory. The discussion is confined to the spectra of 50 stars observed with a resolution of approximately $10 \AA$ over the spectral interval from $1050 \AA$ to $2000 \AA$. Following a qualitative description of the spectra, observed equivalent widths of the $\operatorname{Sirv}(1400 \AA)$ and $\operatorname{CIV}(1550 \AA)$ lines are compared with model atmosphere calculations. The results suggest an upward revision of the stellar temperature scale for stars earlier than B3 V.
\end{abstract}

The Wisconsin instrumentation on the OAO-A2 spacecraft includes an objective grating scanning spectrometer sensitive to the spectral interval from $1050 \AA$ to $2000 \AA$. Spectral scans are accomplished by rotating the grating in discrete $10-\AA$ steps. An Ascop $541 \mathrm{~F}$ photomultiplier, mounted behind a $10-\AA$ slot in the focal plane, is operated in both a pulse-counting and direct-current mode. This paper describes some preliminary results from the data for approximately 50 early-type stars. Repeated scans of many of the objects over intervals of time as great as several months show internal agreement of the order of $2 \%$ for those stars not known to be variable. This is probably a realistic value of the photometric accuracy of the data.

Figure 1 shows the variation of the stronger spectral line features with spectral type for main-sequence stars where the digital counts are plotted as a function of wavelength. The CIV resonance line at $1550 \AA$ is the strongest stellar feature in this spectral region for stars earlier than B0. The SiIv line at $1400 \AA$ and the CIv lines at $1550 \AA$ are equal at type B0.5 V and by B1 the SiIv resonance line dominates. At B3 V SiIV is just detectable and SiIII reaches a maximum. The stellar Lyman- $\alpha$ line and Si III blend give a large absorption at Lyman- $\alpha$ in stars later than B3. The contribution to Lyman- $\alpha$ in earlier types is primarily due to interstellar hydrogen and stellar $\mathrm{Nv}$.

The spectra show a strong luminosity effect for supergiants, which is illustrated at

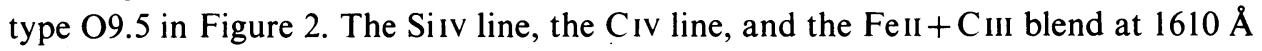
all increase markedly in equivalent width among the supergiants, while maintaining about the same ratio among themselves. These lines display emission components in the red wings of the absorption lines and even at this low resolution the violet displacements of the absorption lines relative to Lyman- $\alpha$ observed by Morton (1967) are apparent. The variations in the strength of Lyman- $\alpha$ shown in the figure are due to variations in the interstellar absorption.

The measured equivalent widths of the CIV and SiIV resonance lines as a function of spectral type are shown in Figure 3. These curves are based on data from approximately 40 stars which show a mean scatter of about $10 \%$. The main sequence and giants have about the same equivalent widths. The supergiants in general have considerably larger equivalent widths as was indicated in Figure 2. For main-sequence 


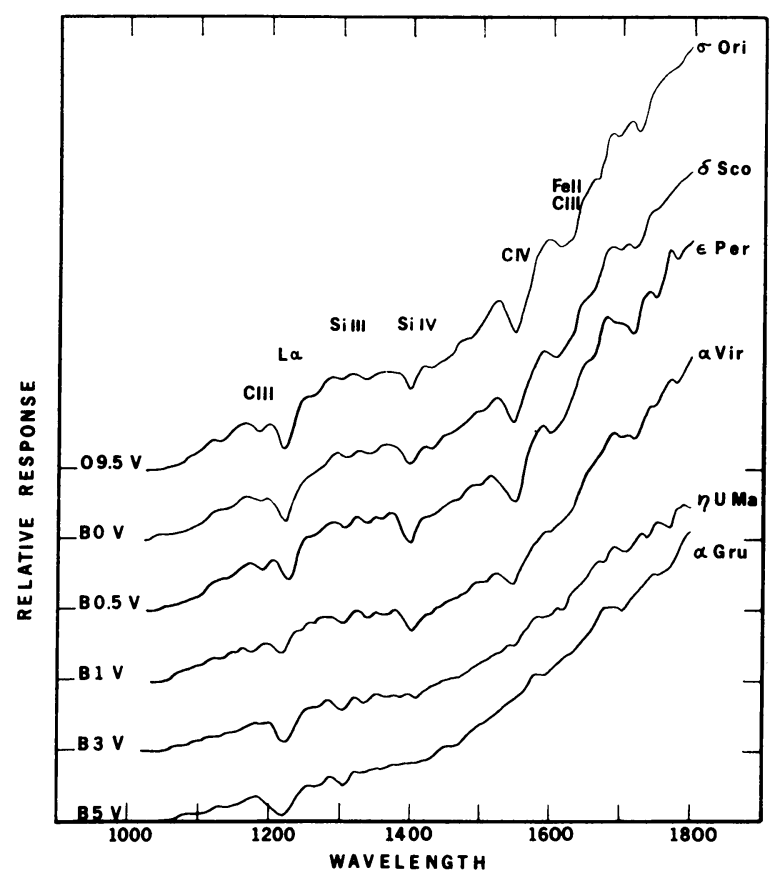

Fig. 1. Spectral scans of early-type stars from $1000-1800 \AA$. The relative response is plotted against wavelength for main-sequence stars of different spectral types.

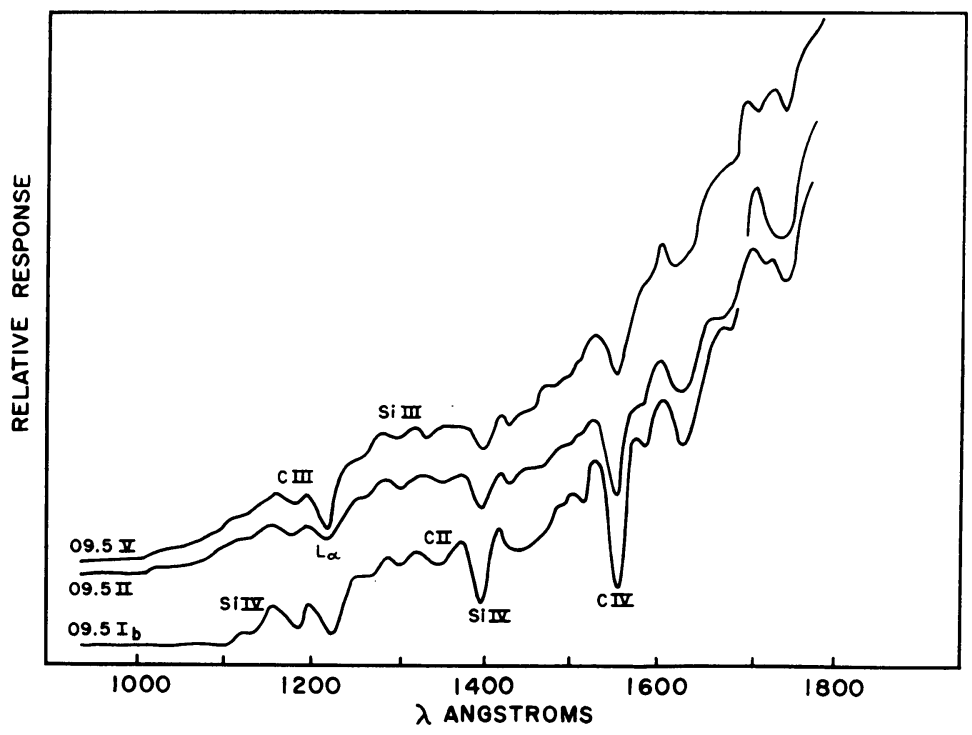

Fig. 2. Spectral scans illustrating the luminosity effect exhibited by the strong stellar lines at spectral type O9.5. 
stars, the CIV line reaches a maximum in the O-type stars, while SIV peaks at about B1. The two lines are of equal strength near B0.5 V.

Some model atmosphere calculations have been carried out for these resonance line profiles. The model atmospheres program was developed by Klinglesmith (1969) and includes hydrogen line blanketing for Lyman and Balmer lines to $n=40$. The line

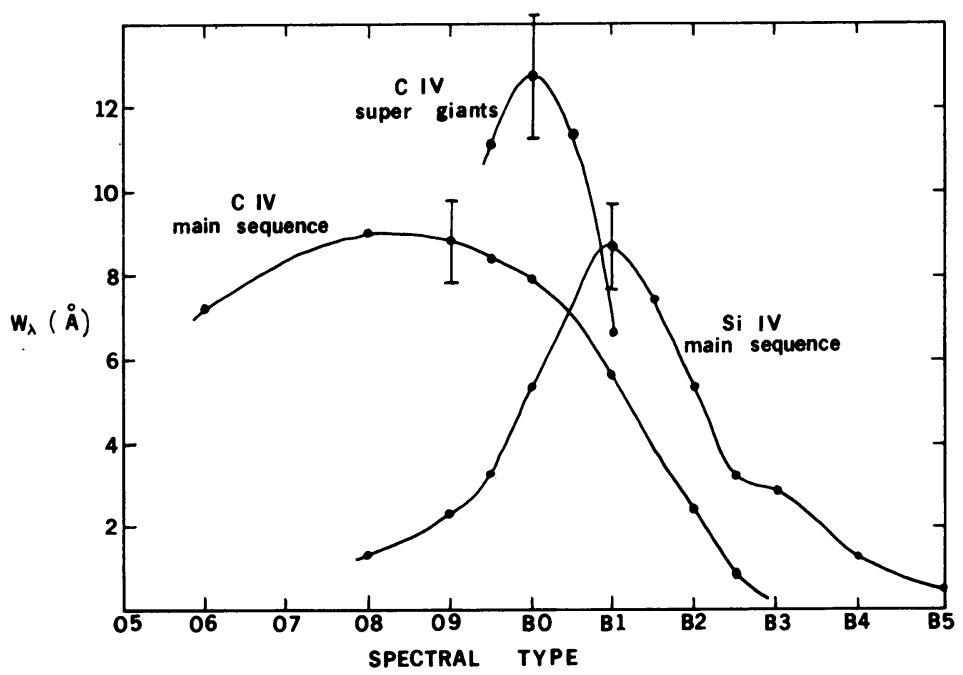

Fig. 3. Mean equivalent widths of the CIV $(1550 \AA)$ and Silv (1400 $\AA)$ lines as a function of spectral type, determined from OAO scans.

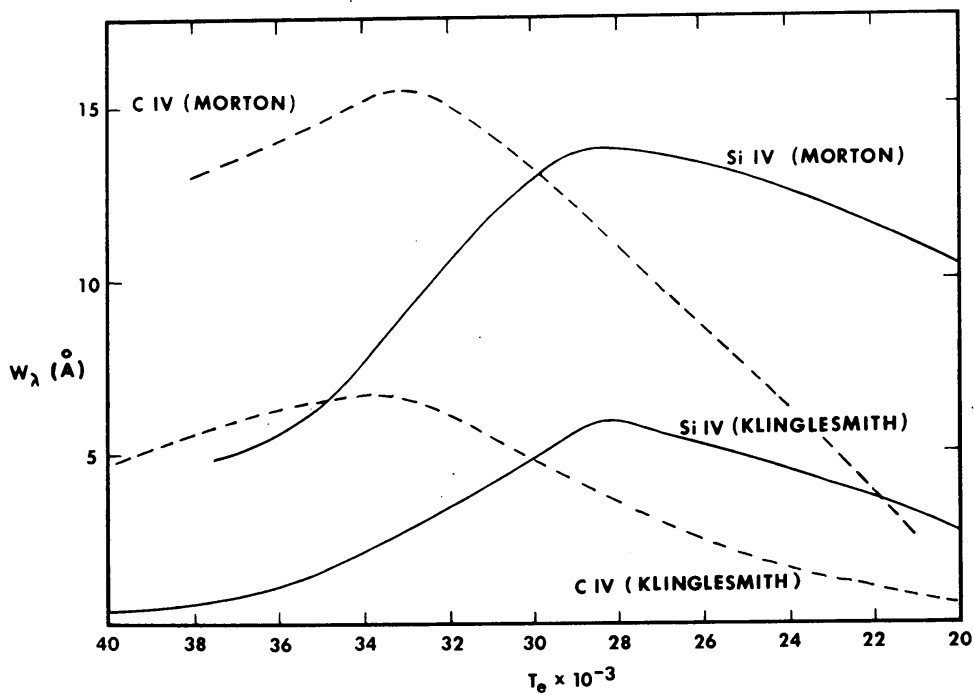

Fig. 4. Theoretical equivalent widths as a function of effective temperature for model atmospheres in local thermodynamic equilibrium for the CIV and SiIV resonance lines. The upper curves are from Bradley and Morton (1969), the lower curves from Klinglesmith (1969). 
broadening mechanisms for the SiIV and CIV lines include electron impact, ion broadening, self-broadening, and radiation damping. The electron collision widths were computed by Oertel on the basis of a revised GKBO theory (Cooper and Oertel, 1967) which gives good agreement for those ions with measured widths. Figure 4 shows the equivalent widths of Civ and SiIV as a function of effective temperature for $\log g=4.0$ models and abundances of $4 \times 10^{-3}$ and $5 \times 10^{-4}$ by weight relative to hydrogen. For comparison, also shown are the equivalent widths determined from the family of models by Morton and associates (cf. Bradley and Morton, 1969) in which a damping constant ten times classical damping was employed, which yields widths about twice those observed. The more detailed calculations yield equivalent widths that are too small by a factor of about 1.5 and would require a factor of 2 increase in abundance. Both sets of curves, however, show the same variations with temperature. The SiIv peaks at about $28000 \mathrm{~K}$ and the $\mathrm{CIV}$ at about $34000 \mathrm{~K}$. These calculations assume LTE, which may not be valid for these lines.

In Figure 5 the observed equivalent widths are plotted against effective temperature using Morton's (1969) most recent temperature scale. The curve is distorted relative to the model atmosphere results, suggesting too large a temperature difference between B0 and B1. The Sirv peaks at $22500 \mathrm{~K}$ in contradiction to the model atmosphere calculations even for unrealistically low values of $\log g$.

A comparison of the continuum fluxes from the OAO spectral scans provides a preliminary temperature scale. If one assumes a temperature of $17800 \mathrm{~K}$ for a B3 V star, then the effective temperatures of a B0.5 V and an $09.5 \mathrm{~V}$ star are about $28500 \mathrm{~K}$ and $33000 \mathrm{~K}$, respectively. These temperatures are in agreement with a temperature scale indicated from the line spectra. Further analysis is currently being carried out for both the continuum fluxes and line strengths measured with the OAO.

Morton's temperature scale for the hotter stars is essentially a Zanstra temperature based on radio emission measures and $\mathrm{H} \alpha$ intensities in diffuse nebulae. This is a mini-

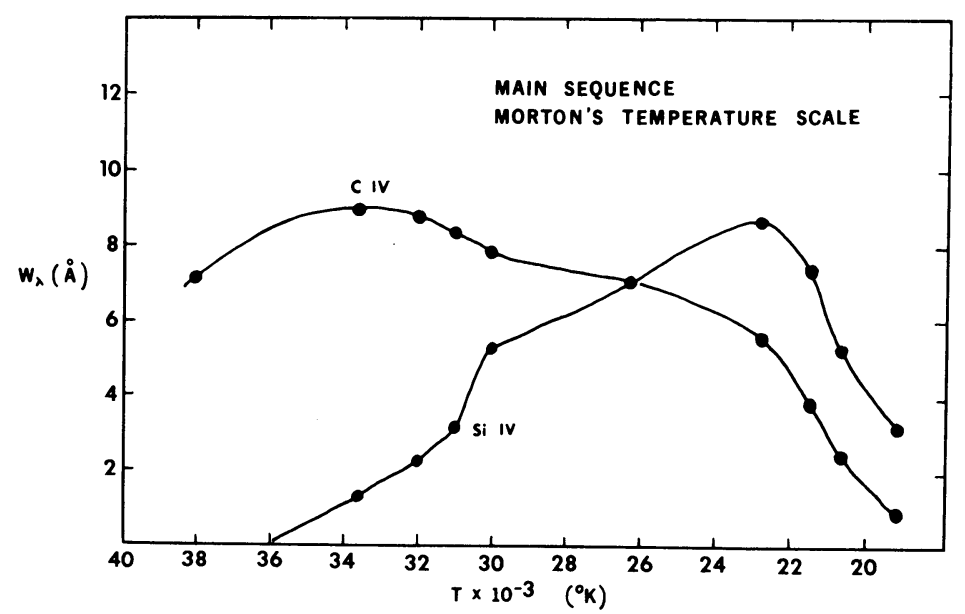

Fig. 5. Observed mean equivalent widths vs. Morton's temperature scale (Morton, 1969). 
mum temperature from the standpoint that some radiation in the Lyman continuum may escape and some ionizing photons will be absorbed by dust grains. In addition, if recent calculations of non-LTE effects by Auer and Mihalas (1969) are valid for the earliest-type stars, higher temperature models must be chosen to obtain sufficient Lyman radiation.

The discussion above is based upon preliminary data reduction of only a part of the OAO spectrophotometry obtained to date. The conclusions presented here are subject to revision according to the results of more extensive analysis.

\section{Acknowledgements}

We wish to thank G. Oertel and D. Klinglesmith for providing, in advance of publication, information on electron collision widths and model atmosphere calculations, respectively.

\section{References}

Auer, L. H. and Mihalas, D.: 1969, Astrophys. J. 156, 681.

Bradley, P. T. and Morton, D. C.: 1969, Astrophys. J. 156, 687.

Cooper, J. and Oertel, G. K.: 1967, Phys. Rev. Letters 18, 985.

Klinglesmith, D.: 1969, private communication.

Morton, D. C.: 1967, Astrophys. J. 147, 1017.

Morton, D. C.: 1969, Astrophys. J. 158, 629.

\section{Discussion}

Carruthers: Are theoretical calculations available for the equivalent width of the $\mathrm{Nv}$ line, which is very strong in the $\mathrm{O}$ stars?

Bless: I know of only those by Morton, which are approximate.

Morton: Your report demonstrates how important it is to have reliable damping constants for the ultraviolet resonance lines.

Bless: Indeed it does. We hope more will be forthcoming for the strong UV lines.

Houziaux: I noticed an interesting luminosity effect for the CIv line dependence vs. spectral type. Did you work any interpretation of this effect?

Bless: No. The problem, of course, is what kind of model atmosphere is appropriate for $\mathrm{O}$ and B-type supergiants.

Müller: Referring to the slide that Bless showed us in which predicted equivalent widths are plotted versus temperature I should like to ask Morton what particular reason he had, which made him increase the damping by a factor of ten in calculating equivalent widths.

Bless: The factor 10 came from an analysis of the solar spectrum by Minnaert and Maldera $(Z$. Astrophys. 2 (1931), 165). In the OB stars the source of broadening must be electrons and protons rather than the van der Waals forces of the hydrogen atoms but one had no better estimates for the broadening of the lines of ionized carbon, nitrogen, silicon etc. when the calculations were started some years ago. 